\title{
复 \\ Análise quantitativa de fatores de receptividade ligados à presença de abrigos de morcegos (Desmodus rotundus) na região centro leste do estado de São Paulo
}

\author{
[Quantitative analysis of receptivity factors related to the presence of (Desmodus rotundus) bat \\ shelters in the central eastern region of the state of São Paulo]
}

\section{"Artigo Científico/Scientific Article"}

\author{
Paulo Jacques Mialhe*, Luiz Eduardo Moschini
}

Departamento de Ciências Ambientais, Universidade Federal de São Carlos, São Carlos-SP, Brasil.

*Autor para correspondência/Corresponding author: E-mail: paulomialhe@gmail.com

\begin{abstract}
Resumo
Durante os anos de 2002 a 2004 foi realizado estudo na região centro leste do estado de São Paulo, com objetivo de analisar quantitativamente determinantes de receptividade à presença de abrigos de morcegos hematófagos Desmodus rotundus (E. Geoffroy, 1810, Chiroptera, Phyllostomidae) utilizando de Sistema de Informações Geográficas (SIG). Foram encontrados 1.566 D. rotundus distribuídos em 93 abrigos em 18 municípios. Em relação à topografia, a maioria dos abrigos artificiais estavam localizados em áreas de baixas declividades e em cotas altimétricas inferiores a $712 \mathrm{~m}$, porém a maioria dos abrigos naturais estavam localizados em cotas altimétricas entre $712-812 \mathrm{~m}$ podendo ser um indício de altitudes preferenciais à colonização de $D$. rotundus para esse tipo de abrigo. Em relação à cobertura e ao uso do solo, a maioria dos abrigos estavam em área de agricultura e nenhum abrigo natural foi encontrado em áreas de formação florestal, indicando alto grau de antropização do meio rural e florestal da região, com aumento da oferta de abrigos artificiais para $D$. rotundus em relação aos abrigos naturais. A quantificação dos fatores de receptividade relacionados à topografia e ao tipo e cobertura do solo podem auxiliar na elaboração de modelos de áreas de risco de ataques de morcegos hematófagos a herbívoros domésticos, devendo-se levar em consideração os efeitos de escala de cada trabalho e as características de paisagem da região estudada.
\end{abstract}

Palavras-chave: morcego hematófago; abrigos; SIG.

\begin{abstract}
From 2002 to 2004 a study was carried out in the central eastern region of the state of São Paulo, with the objective of quantitatively analyzing determinants of receptivity to the presence of shelters of vampire bats Desmodus rotundus (E. Geoffroy, 1810, Chiroptera, Phyllostomidae). In total, 1566 D. rotundus were found distributed among 93 shelters in 18 municipalities. Regarding the topography, most of the artificial shelters were located in areas with low slopes and at altitudes below $712 \mathrm{~m}$, but most natural shelters were located at altitudes between $712-812 \mathrm{~m}$, which may be an indication of preferential altitudes to colonization of $D$. rotundus for this type of shelter. Regarding the coverage and use of the soil, most shelters were in the agricultural area and no natural shelter was found in areas of forest formation, indicating a high degree of anthropization of the rural and forest environment in the region, with an increase in the supply of shelters for D. rotundus compared to natural shelters. The quantification of receptivity factors related to topography, soil type and cover could help with the development of models of areas at risk for attacks by vampire bats on domestic livestock, taking into account the effects of the scale of each study and the landscape characteristics of the region studied.
\end{abstract}

Keywords: vampire bat; shelters; GIS. 


\section{Introdução}

O morcego hematófago Desmodus rotundus

(E. Geoffroy, 1810), pertence à família Phyllostomidae e ordem Chiroptera (Neuweiler, 2000), é a espécie mais comum e abundante de morcego hematófago, alimentando-se exclusivamente de sangue de mamíferos, sendo encontrado somente no Novo Mundo, ocorrendo desde o norte do México até o norte da Argentina e Uruguai (Greenhall, 1983). Habita áreas florestadas assim como regiões desérticas, abrigando-se em locais seguros contra predadores, podendo ser naturais ou artificiais. Entre os abrigos naturais existem as cavernas, furnas, oco de árvores, e entre abrigos artificiais bueiros, porão ou sótão de casas, cisternas e outras construções desabitadas com ambientes escuros e protegidos (Greenhall, 1983; BRASIL, 2009), sendo comumente encontrado em habitats antropogênicos (Johnson et al., 2014).

Devido ao seu papel na transmissão de doenças de caráter zoonótico, como a raiva, para o gado e seres humanos na região Neotropical, $D$. rotundus tem recebido atenção especial das autoridades de saúde pública em toda América Latina (Vigilato et al., 2013).

A raiva paralítica bovina representa um dos principais problemas para a pecuária nas regiões tropicais das Américas (Lee et al., 2012). A raiva é causada por um vírus do gênero Lyssavirus (família Rhabdoviridae), acomete um grande número de mamíferos, ocasionando uma encefalomielite de curso agudo e letal (Fisher et al., 2018). O vírus está presente na saliva de animais raivosos e, consequentemente, a transmissão ocorre, em geral, através da mordedura (Acha e Szyfres, 2003).

$\mathrm{Na}$ natureza, o vírus da raiva é mantido por ciclos ocasionalmente inter-relacionados, denominados ciclos aéreo, urbano, rural e silvestre. Ciclo aéreo refere-se à raiva em morcegos, sendo os demais ciclos denominados ciclos terrestres. Ciclo urbano refere-se à raiva em cães e gatos domésticos; ciclo rural refere-se à raiva dos herbívoros, que envolve principalmente bovinos e equinos e na qual o principal vetor é o morcego hematófago $D$. rotundus. O ciclo silvestre refere-se à raiva associada a espécies silvestres, sendo por vezes utilizado englobando o ciclo aéreo (Batista et al., 2007).

Estudos para o controle dessa enfermidade vêm sendo direcionados paralelamente aos de comportamento, controle e dinâmica de populações do morcego $D$. rotundus $\mathrm{e}$ consequentemente de aspectos geográficos que favorecem a existência de abrigos em certas áreas, em detrimento de outras (Gomes et al., 2007).

Embora a transformação de paisagens pela atividade humana possa afetar negativamente as populações de várias espécies de morcegos, outras espécies podem se beneficiar dessas transformações. Uma dessas espécies é o morcego hematófago $D$. rotundus, que muitas vezes pode ser encontrado em paisagens dominadas por atividades pecuárias (Bolívar-Cimé et al., 2019).

Entende-se por receptividade a interrelação do conjunto de variáveis que expressam a capacidade de manutenção e que permitem a difusão do transmissor do vírus da raiva nos herbívoros domésticos, o morcego hematófago $D$. rotundus. Essas variáveis são: presença de bovinos, tipo de criação (intensiva ou extensiva), ocorrência de afloramentos de rocha calcária, declividade do terreno, ocorrência de matas permanentes, localização de abrigos naturais permanentes e temporários, localização de abrigos artificiais e de edificações com potencial utilização como abrigos para o transmissor (BRASIL, 2009; Dias et al., 2011).

Em referência ao uso e cobertura da terra, o estado de São Paulo apresenta diferentes tipos de usos e cobertura do início da sua ocupação, com incremento paulatino das áreas de cana-de-açúcar em parcelas do estado (Gomes al., 2011). Essas alterações na paisagem podem influenciar a distribuição de abrigos de $D$. rotundus e a transmissão do vírus da raiva. A maioria dos abrigos de $D$. rotundus encontrados no estado de São Paulo são artificiais, sendo estes mais comuns na região oeste enquanto os abrigos naturais são mais abundantes na região leste do estado, onde há maior predominância da Floresta Atlântica e de topografia acidentada (Gomes e Uieda, 2004; CDA-SP, 2016;).

Existem poucos estudos quantitativos dos determinantes de receptividade relacionados à disponibilidade de abrigos aptos para $D$. rotundus, sendo a maioria deles qualitativos. Este trabalho teve como objetivo analisar quantitativamente os determinantes de receptividade à presença de abrigos aptos de morcegos hematófagos $D$. rotundus na região centro leste do estado de São Paulo, utilizando de Sistema de Informações Geográficas. 


\section{Material e Métodos}

\section{Área de estudo}

A pesquisa foi realizada durante os anos de 2002 a 2004 na zona rural de 18 municípios localizados na região centro leste do estado de São Paulo, abrangendo a Região Geográfica Intermediária de Araraquara (municípios de Américo Brasiliense, Tabatinga, Cândido Rodrigues, Araraquara, Descalvado, Trabiju, Boa Esperança do Sul, Dobrada, Matão, Santa Lucia, Taquaritinga), a Região Geográfica Intermediária de São Carlos (municípios de São Carlos, Ribeirão Bonito, Ibaté, Dourado, Descalvado) além do município de Analândia, localizado na Região Geográfica Intermediária de Rio Claro (Figura 1).

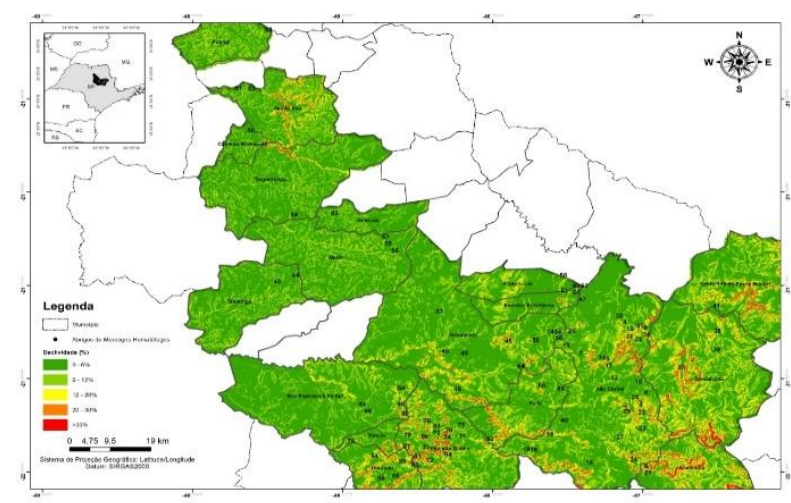

Figura 1. Mapa da declividade da região em estudo e abrigos de morcegos hematófagos (Desmodus rotundus) encontrados.

A área de estudo apresenta predominantemente baixa declividade e cotas altimétricas entre 412 - 1012 m, com imóveis rurais de tamanhos na sua maioria entre 50 e 500 hectares, onde ocorre a pratica da pecuária leiteira e de corte, aves, ovinos e culturas como cana-deaçúcar, soja, laranja, hortaliças e pequenas lavouras de subsistência (IEA, 2002)

A região oferece boas condições para a formação de abrigos de $D$. rotundus, tais como construções abandonadas, grutas, viadutos, túneis e temperatura favoráveis, assim como recursos (água e alimento) em abundância.

\section{Metodologia}

Durante os anos de 2002 a 2004 foram realizadas na zona rural dos municípios em estudo buscas por colônias de morcegos hematófagos em abrigos naturais e artificiais, seguindo informações coletadas de proprietários e funcionários rurais sobre a presença de abrigos destes morcegos na região. Quando localizados, os abrigos foram georreferenciados com o uso de um receptor GPS (Global Positioning System) modelo Garmim etrex ${ }^{\circledR}$ e estimada a população de morcegos existentes nos abrigos.

A população de $D$. rotundus nos abrigos foi estimada por observação visual do número de indivíduos em cada abrigo através de contagem simples de indivíduos, e quando encontradas colônias populosas contou-se a quantidade de morcegos aglomerados por metro quadrado e multiplicou-se pelo total da área ocupada pelos mesmos no abrigo (Mialhe e Moschini, 2018), além do volume de fezes destes morcegos existentes no chão, paredes ou outras estruturas. Elas se caracterizam por serem negras em pequenas gotas (quando há poucos indivíduos no local) ou então manchas escorridas e poças de fezes (quando há muitos) (Oliveira et al., 2009).

Pode-se diferenciar se esse é um abrigo ativo, ou seja, se naquele momento há a presença de morcegos hematófagos; ou inativo, se já houve essa presença. Nos abrigos ativos, as fezes são bem escuras, brilhantes e em estado pastoso, assemelhando-se a gotas de óleo queimado. Nos abrigos inativos, essas fezes estarão opacas e secas. O odor amoniacal de sangue digerido é característico nesses locais (Bredt, 1996; Brasil, 2009).

A partir das coordenadas geográficas obtidas no georreferenciamento dos abrigos, foram criados mapas temáticos relacionados à declividade, altitude, uso e cobertura do solo, utilizando-se Cartas Topográficas do IBGE na escala 1:50.000 e o software ARCGIS ${ }^{\circledR}$ 10.2.2.

\section{Resultados}

Foram encontrados 1.566 indivíduos da espécie D. rotundus distribuídos em 93 abrigos nos municípios estudados, sendo a maioria artificiais (87 abrigos, 93,55\%) e seis abrigos naturais $(6,45 \%)$, conforme ilustram as Tabelas 1 a 5. O número de D. rotundus encontrados nos abrigos variou entre 1 a 374 morcegos.

Em relação a declividade, a maioria dos abrigos (44,08\%) estavam em áreas com declividade de até $6 \%$, e apenas dois abrigos $(2,15 \%)$, sendo um abrigo artificial outro natural que estavam em áreas com declividade e acima de $30 \%$, conforme ilustra a Tabela 6.

A Figura 1 mostra o mapa da declividade da região em estudo e os abrigos de $D$. rotundus encontrados. 
Tabela 1. Localização, tipos de abrigos, população e cobertura vegetal de abrigos de Desmodus rotundus encontrados na Região Geográfica Intermediaria de Araraquara (municípios de Américo Brasiliense e Araraquara).

\begin{tabular}{|c|c|c|c|c|c|c|}
\hline ID & Coordenada $Y$ & Coordenada X & Município & Tipo de abrigo & População & $\begin{array}{l}\text { Cobertura } \\
\text { vegetal }\end{array}$ \\
\hline 60 & $-21,6875$ & $-47,9361$ & Américo Brasiliense & TU & 4 & $\mathrm{AG}$ \\
\hline 61 & $-21,6988$ & $-47,9605$ & Américo Brasiliense & $\mathrm{CAB}$ & 2 & $\mathrm{AG}$ \\
\hline 62 & $-21,6875$ & $-47,9188$ & Américo Brasiliense & $\mathrm{TU}$ & 1 & $\mathrm{AG}$ \\
\hline 63 & $-21,6877$ & $-47,9361$ & Américo Brasiliense & TU & 2 & $\mathrm{AG}$ \\
\hline 47 & $-21,7022$ & $-47,9230$ & Américo Brasiliense & $\mathrm{CAB}$ & 7 & CSP \\
\hline 48 & $-21,8413$ & $-48,2125$ & Araraquara & $\mathrm{CAB}$ & 7 & AG \\
\hline 64 & $-21,8766$ & $-48,0522$ & Araraquara & $\mathrm{M}$ & 78 & $\mathrm{AG}$ \\
\hline 67 & $-21,7483$ & $-48,2241$ & Araraquara & $\mathrm{CAB}$ & 4 & $\mathrm{AG}$ \\
\hline 14 & $-21,7955$ & $-47,9747$ & Araraquara & $\mathrm{CAB}$ & 4 & CSP \\
\hline 16 & $-21,7955$ & $-47,9727$ & Araraquara & $\mathrm{CAB}$ & 15 & CSP \\
\hline 34 & $-21,7958$ & $-47,9752$ & Araraquara & $\mathrm{CA}$ & 0 & CSP \\
\hline 49 & $-21,8461$ & $-48,1719$ & Araraquara & GP & 22 & CSP \\
\hline 45 & $-21,8177$ & $-48,0791$ & Araraquara & TU & 2 & FF \\
\hline 46 & $-21,9305$ & $-48,1866$ & Araraquara & TU & 2 & $\mathrm{FF}$ \\
\hline 59 & $-21,8155$ & $-48,0211$ & Araraquara & TU & 8 & $\mathrm{FF}$ \\
\hline & \multicolumn{4}{|c|}{ Total D. rotundus } & \multicolumn{2}{|l|}{158} \\
\hline
\end{tabular}

Tabela 2. Localização, tipos de abrigos, população e cobertura vegetal de abrigos de Desmodus rotundus encontrados na Região Geográfica Intermediaria de Araraquara (municípios de Boa Esperança do Sul, Cândido Rodrigues, Descalvado, Dobrada, Matão, Santa Lucia, Tabatinga, Taquaritinga e Trabiju).

\begin{tabular}{lllllll}
\hline ID & Coordenada Y & Coordenada X & Município & Tipo de abrigo & População & $\begin{array}{l}\text { Cobertura } \\
\text { vegetal }\end{array}$ \\
\hline 80 & $-21,9872$ & $-48,2972$ & Boa Esperança do Sul & CAB & 8 & AG \\
89 & $-21,9780$ & $-48,3758$ & Boa Esperança do Sul & PO & 7 & AG \\
91 & $-21,9666$ & $-48,3872$ & Boa Esperança do Sul & CAB & 2 & AG \\
88 & $-21,9283$ & $-48,3052$ & Boa Esperança do Sul & CAB & 4 & CSP \\
90 & $-21,9655$ & $-48,3063$ & Boa Esperança do Sul & CR & 3 & PAS \\
50 & $-21,3233$ & $-48,6225$ & Cândido Rodrigues & TU & 4 & AG \\
37 & $-21,8791$ & $-47,7144$ & Descalvado & TL & 6 & AG \\
38 & $-21,7936$ & $-47,6380$ & Descalvado & PO & 6 & CSP \\
41 & $-21,7341$ & $-47,6402$ & Descalvado & CA & 21 & CSP \\
39 & $-21,8372$ & $-47,6397$ & Descalvado & TU & 70 & FF \\
42 & $-21,5172$ & $-48,4461$ & Dobrada & TL & 3 & CSP \\
85 & $-22,1347$ & $-48,3172$ & Dourado & CAB & PAS \\
55 & $-21,5727$ & $-48,3333$ & Matão & TL & 8 & AG \\
56 & $-21,6052$ & $-48,3188$ & Matão & CAB & 2 & AG \\
57 & $-21,5727$ & $-48,3377$ & Matão & CAB & 9 & AG \\
58 & $-21,6625$ & $-47,9630$ & Santa Lúcia & CAB & 2 & AG \\
43 & $-21,6772$ & $-48,5669$ & Tabatinga & TU & 2 & AG \\
44 & $-21,6633$ & $-48,5280$ & Tabatinga & Taquaritinga & & CSP \\
54 & $-21,5207$ & $-48,5311$ & Trabijú & FF & FF \\
78 & $-22,0525$ & $-48,4111$ & & 13 &
\end{tabular}

$\mathrm{ID}=$ identificação, $\mathrm{A}=$ artificial, $\mathrm{N}=$ natural, $\mathrm{MT}=$ maternidade, $\mathrm{MS}=$ abrigo de macho solteiro, $\mathrm{D}=$ digestório, $\mathrm{C}=\mathrm{casa}, \mathrm{CAB}=$ casa abandonada, $\mathrm{CX}=$ caixa de agua abandonada, $\mathrm{TU}=$ túnel, $\mathrm{G}=$ gruta, $\mathrm{PO}=$ porão, $\mathrm{TL}=$ tulha, $\mathrm{GP}=$ galeria de agua pluvial, $\mathrm{CR}$ $=$ curral, $\mathrm{M}=$ moinho, $\mathrm{AG}=$ Agricultura Perene, $\mathrm{CSP}=$ Cultura Semi Perene, $\mathrm{FF}=$ Formação Florestal, $\mathrm{PAS}=$ Pastagens .

Em relação à altitude, a maioria dos abrigos artificiais (68 abrigos, $78,16 \%)$ estavam localizados em cotas altimétricas inferiores a 712 $\mathrm{m}$, com predominância de abrigos localizados em cotas altimétricas entre 512 - 712 m (66 abrigos, $75,86 \%)$, conforme ilustra a Tabela 7 .
A maioria dos abrigos naturais (4 abrigos, $66,67 \%)$ estavam localizados em cotas altimétricas entre $712-812 \mathrm{~m}$. Apesar de terem sido encontrado um abrigo natural de $D$. rotundus $(16,66 \%)$ em cota inferior a $512 \mathrm{~m}$ e um abrigo natural $(16,66 \%)$ em cota entre $912-1012 \mathrm{~m}$, nenhum abrigo natural de morcego hematófago 
foi encontrado na cota altimétrica entre $512-712$ $\mathrm{m}$, conforme ilustra a Tabela 7.

A Figura 2 mostra o mapa altimétrico da região em estudo e os abrigos de D. rotundus encontrados.

Em relação à cobertura e uso do solo, 51 $(54,83 \%)$ do total de abrigos estavam em área de agricultura, $15(16,12 \%)$ em áreas de cultura semi perene, $16(17,20 \%)$ em áreas de formação florestal e $11(11,82 \%)$ em áreas de pastagens.

A Figura 3 mostra o mapa do uso e cobertura do solo da região em estudo e os abrigos de D. rotundus encontrados.

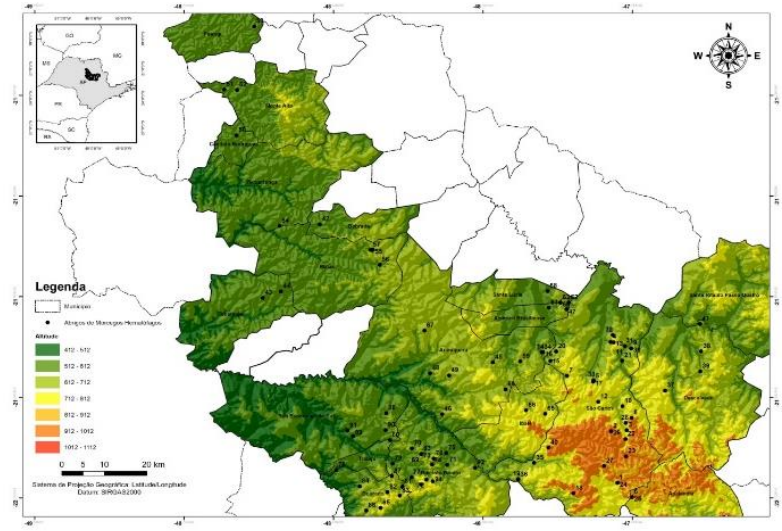

Figura 2. Mapa altimétrico da região em estudo e abrigos de morcegos hematófagos (Desmodus rotundus) encontrados.

Tabela 3. Localização, tipos de abrigos, população e cobertura vegetal de abrigos de Desmodus rotundus encontrados na Região Geográfica Intermediaria de São Carlos (municípios de Descalvado, Dourado e Ibaté) e Região Geográfica Intermediaria de Rio Claro, município de Analândia.

\begin{tabular}{lllllll}
\hline ID & Coordenada Y & Coordenada X & Município & Tipo de abrigo & População & $\begin{array}{l}\text { Cobertura } \\
\text { vegetal }\end{array}$ \\
\hline 37 & $-21,8791$ & $-47,7144$ & Descalvado & TL & 6 & AG \\
38 & $-21,7936$ & $-47,6380$ & Descalvado & PO & 6 & CSP \\
41 & $-21,7341$ & $-47,6402$ & Descalvado & CA & CA & CSP \\
39 & $-21,8372$ & $-47,6397$ & Descalvado & CAB & 34 & FF \\
83 & $-22,1077$ & $-48,2761$ & Dourado & TL & 21 & AG \\
86 & $-22,1405$ & $-48,3475$ & Dourado & CAB & 2 & AG \\
87 & $-22,0655$ & $-48,2930$ & Dourado & CAB & 2 & AG \\
82 & $-22,1002$ & $-48,3027$ & Dourado & TL & 11 & CSP \\
84 & $-22,0875$ & $-48,3613$ & Dourado & TL & 3 & PAS \\
85 & $-22,1347$ & $-48,3172$ & Dourado & CAB & PAS \\
66 & $-21,9219$ & $-48,0088$ & Ibaté & GR & 5 & AG \\
65 & $-21,9297$ & $-47,9683$ & Ibaté & CA & 12 & PAS \\
6 & $-22,1113$ & $-47,7844$ & $-47,7844$ & Analândia & & AG \\
25 & $-22,1113$ & & & & AG
\end{tabular}

Total D. rotundus

135

$\mathrm{ID}=$ identificação, $\mathrm{A}=$ artificial, $\mathrm{N}=$ natural, $\mathrm{MT}=$ maternidade, $\mathrm{MS}=$ abrigo de macho solteiro, $\mathrm{D}=$ digestório, $\mathrm{C}=$ casa, $\mathrm{CAB}=$ casa abandonada, $\mathrm{CX}=$ caixa de agua abandonada, $\mathrm{TU}=$ túnel, $\mathrm{G}=$ gruta, $\mathrm{PO}=$ porão, $\mathrm{TL}=$ tulha, $\mathrm{GP}=$ galeria de agua pluvial, $\mathrm{CR}$ = curral, $\mathrm{M}=$ moinho, $\mathrm{AG}=$ Agricultura Perene, $\mathrm{CSP}=$ Cultura Semi Perene, $\mathrm{FF}=$ Formação Florestal, $\mathrm{PAS}=$ Pastagens .

Tabela 4. Localização, tipos de abrigos, população e cobertura vegetal de abrigos de Desmodus rotundus encontrados na Região Geográfica Intermediaria de São Carlos (município de Ribeirão Bonito).

\begin{tabular}{|c|c|c|c|c|c|c|}
\hline ID & Coordenada Y & Coordenada $X$ & Município & Tipo de abrigo & População & $\begin{array}{l}\text { Cobertura } \\
\text { vegetal }\end{array}$ \\
\hline 68 & $-22,0836$ & $-48,2313$ & Ribeirão Bonito & $\mathrm{CAB}$ & 12 & $\mathrm{AG}$ \\
\hline 70 & $-22,0052$ & $-48,2508$ & Ribeirão Bonito & GR & 3 & AG \\
\hline 71 & $-22,0411$ & $-48,1761$ & Ribeirão Bonito & $\mathrm{CAB}$ & 2 & $\mathrm{AG}$ \\
\hline 72 & $-22,0730$ & $-48,2200$ & Ribeirão Bonito & $\mathrm{CAB}$ & 2 & $\mathrm{AG}$ \\
\hline 79 & $-22,0383$ & $-48,2919$ & Ribeirão Bonito & $\mathrm{CAB}$ & 14 & CSP \\
\hline 94 & $-22,0768$ & $-48,2064$ & Ribeirão Bonito & GR & 7 & CSP \\
\hline 69 & $-22,0175$ & $-48,2311$ & Ribeirão Bonito & TL & 71 & $\mathrm{FF}$ \\
\hline 73 & $-22,0177$ & $-48,2308$ & Ribeirão Bonito & $\mathrm{TU}$ & 26 & $\mathrm{FF}$ \\
\hline 76 & $-22,0269$ & $-48,2047$ & Ribeirão Bonito & TU & 12 & $\mathrm{FF}$ \\
\hline 92 & $-22,0473$ & $-48,1168$ & Ribeirão Bonito & $\mathrm{TU}$ & 124 & $\mathrm{FF}$ \\
\hline 93 & $-22,0171$ & $-48,2304$ & Ribeirão Bonito & TU & 35 & FF \\
\hline 74 & $-22,0402$ & $-48,2077$ & Ribeirão Bonito & TU & 3 & PAS \\
\hline 75 & $-22,0152$ & $-48,1783$ & Ribeirão Bonito & GR & 1 & PAS \\
\hline 77 & $-22,0680$ & $-48,2513$ & Ribeirão Bonito & $\mathrm{CAB}$ & 2 & PAS \\
\hline 81 & $-22,0883$ & $-48,2708$ & Ribeirão Bonito & $\mathrm{CAB}$ & 5 & PAS \\
\hline & \multicolumn{4}{|c|}{ Total D. rotundus } & 319 & \\
\hline
\end{tabular}




\section{Discussão}

A região em estudo é composta por declividade predominantemente inferior a $6 \%$, o que refletiu na maioria dos abrigos localizados (41 abrigos, 44,08\%) que estavam nesta cota de declividade e somente dois abrigos $(2,15 \%)$, um natural e outro artificial, com declividade acima de $30 \%$. Esses valores são muito inferiores aos encontrados a outros estudos, que apresentam uma tendência para localização dos abrigos em áreas de declividade acentuada, acima de $40 \%$ (Silva et al., 2001).

A cotas altimétricas dos municípios estudados apresentaram na sua maior parte cotas inferiores a $712 \mathrm{~m}$ (Figura 2), o que repercutiu na maioria dos abrigos artificiais localizados. Porém observou-se uma diferença nas cotas altimétricas entre abrigos artificiais e naturais: enquanto que a maioria dos abrigos artificiais (66 abrigos, $75,86 \%$ ) estavam localizados em cotas altimétricas entre 512 - $712 \mathrm{~m}$, nenhum abrigo natural estava nesta cota. Os abrigos naturais encontravam-se na sua maioria (4 abrigos, $66,67 \%$ ) localizados em cotas altimétricas entre $712-812 \mathrm{~m}$, enquanto que somente $12,65 \%$ dos abrigos artificiais encontravam-se nesta cota altimétrica (Tabela 7).
Cotas altimétricas semelhantes às encontradas no presente estudo para abrigos naturais também foram relatadas na região cárstica de Minas Gerais, de declividade acentuada, onde a totalidade dos abrigos naturais estavam em cotas acima de $712 \mathrm{~m}$, com predominância $(63,63 \%)$ de cotas entre 710 - $810 \mathrm{~m}$ (Almeida, 2002), percentual próximo ao encontrado para cotas altimétricas de abrigos naturais no presente estudo $(66,67 \%)$.

Aspectos geográficos favorecem a existência de diferentes tipos de abrigos em certas áreas, em detrimento de outras. Em estudo na região cárstica de Cordisburgo - MG (Almeida, 2002), foram encontrados 18 abrigos naturais e nenhum abrigo artificial foi colonizado por $D$. rotundus. No presente estudo, realizado em região com topografia predominantemente de baixa declividade, a maioria dos abrigos (87 abrigos, $93,55 \%$ ) eram artificiais, assim como a maioria dos abrigos de $D$. rotundus encontrados no estado de São Paulo (Gomes e Uieda, 2004; CDA-SP, 2016), indicando alta adaptabilidade de $D$. rotundus a ambientes antropizados.

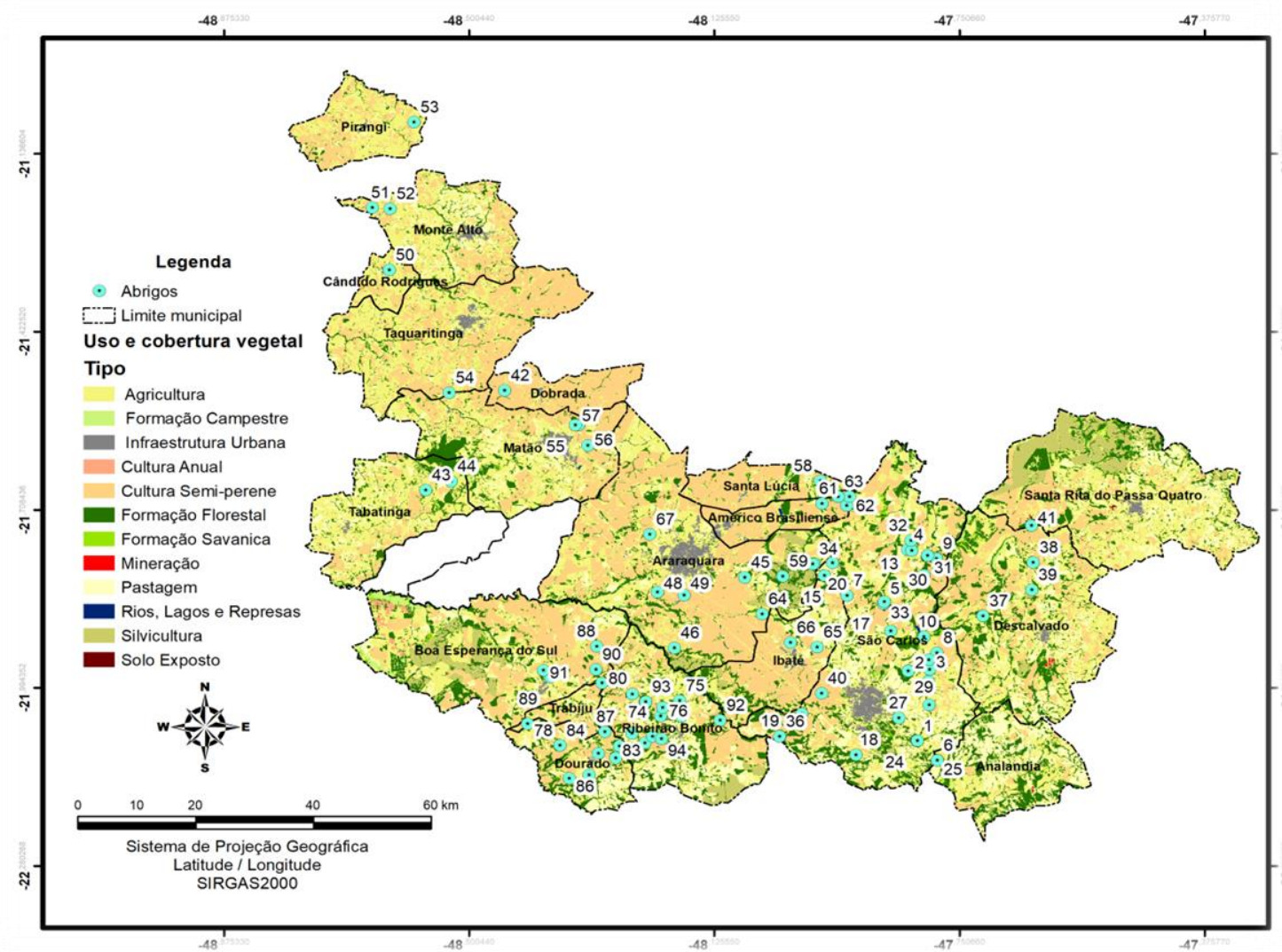

Figura 3. Mapa da cobertura e uso do solo da região em estudo e abrigos de morcegos hematófagos (Desmodus rotundus). 
Tabela 5. Localização, tipos de abrigos, população e cobertura vegetal de abrigos de Desmodus rotundus encontrados na Região Geográfica Intermediaria de São Carlos (município de São Carlos).

\begin{tabular}{|c|c|c|c|c|c|c|}
\hline ID & Coordenada $\mathbf{Y}$ & Coordenada $X$ & Município & Tipo de abrigo & População & $\begin{array}{l}\text { Cobertura } \\
\text { vegetal }\end{array}$ \\
\hline 1 & $-22,0802$ & $-47,8155$ & São Carlos & TL & 68 & $\mathrm{AG}$ \\
\hline 2 & $-21,9675$ & $-47,8286$ & São Carlos & $\mathrm{CA}$ & 13 & $\mathrm{AG}$ \\
\hline 3 & $-21,9652$ & $-47,7961$ & São Carlos & $\mathrm{CX}$ & 9 & $\mathrm{AG}$ \\
\hline 4 & $-21,7733$ & $-47,8297$ & São Carlos & TU & 43 & $\mathrm{AG}$ \\
\hline 5 & $-21,8594$ & $-47,8661$ & São Carlos & TL & 12 & $\mathrm{AG}$ \\
\hline 7 & $-21,8470$ & $-47,9222$ & São Carlos & $\mathrm{PO}$ & 9 & $\mathrm{AG}$ \\
\hline 8 & $-21,9386$ & $-47,7852$ & São Carlos & GR & 374 & $\mathrm{AG}$ \\
\hline 9 & $-21,7872$ & $-47,7861$ & São Carlos & TL & 37 & $\mathrm{AG}$ \\
\hline 10 & $-21,9130$ & $-47,8047$ & São Carlos & $\mathrm{CAB}$ & 2 & $\mathrm{AG}$ \\
\hline 12 & $-21,9036$ & $-47,8558$ & São Carlos & $\mathrm{CAB}$ & 11 & $\mathrm{AG}$ \\
\hline 13 & $-21,7744$ & $-47,8233$ & São Carlos & TU & 5 & $\mathrm{AG}$ \\
\hline 17 & $-21,8597$ & $-47,8669$ & São Carlos & $\mathrm{TL}$ & 12 & $\mathrm{AG}$ \\
\hline 19 & $-22,0722$ & $-48,0252$ & São Carlos & $\mathrm{TL}$ & 6 & $\mathrm{AG}$ \\
\hline 20 & $-21,7941$ & $-47,9450$ & São Carlos & $\mathrm{CAB}$ & 4 & $\mathrm{AG}$ \\
\hline 21 & $-21,8141$ & $-47,8050$ & São Carlos & $\mathrm{TU}$ & 7 & $\mathrm{AG}$ \\
\hline 22 & $-21,9847$ & $-47,7980$ & São Carlos & $\mathrm{CX}$ & 74 & $\mathrm{AG}$ \\
\hline 23 & $-22,0222$ & $-47,7969$ & São Carlos & $\mathrm{CX}$ & 3 & $\mathrm{AG}$ \\
\hline 24 & $-22,0800$ & $-47,8150$ & São Carlos & $\mathrm{TL}$ & 3 & $\mathrm{AG}$ \\
\hline 27 & $-22,0433$ & $-47,8436$ & São Carlos & $\mathrm{CA}$ & 1 & $\mathrm{AG}$ \\
\hline 28 & $-21,9497$ & $-47,7977$ & São Carlos & CAB & 14 & $\mathrm{AG}$ \\
\hline 29 & $-21,9677$ & $-47,8288$ & São Carlos & $\mathrm{TL}$ & 1 & $\mathrm{AG}$ \\
\hline 30 & $-21,7872$ & $-47,7861$ & São Carlos & $\mathrm{TL}$ & 4 & $\mathrm{AG}$ \\
\hline 36 & $-22,0730$ & $-48,0255$ & São Carlos & $\mathrm{TL}$ & 3 & $\mathrm{AG}$ \\
\hline 40 & $-22,0038$ & $-47,9613$ & São Carlos & $\mathrm{TL}$ & 22 & CSP \\
\hline 11 & $-21,7822$ & $-47,7994$ & São Carlos & $\mathrm{TU}$ & 4 & $\mathrm{FF}$ \\
\hline 18 & $-22,1027$ & $-47,9086$ & São Carlos & $\mathrm{CAB}$ & 2 & $\mathrm{FF}$ \\
\hline 31 & $-21,7822$ & $-47,7994$ & São Carlos & TU & 3 & $\mathrm{FF}$ \\
\hline 33 & $-21,8566$ & $-47,8658$ & São Carlos & $\mathrm{PO}$ & 2 & FF \\
\hline 35 & $-22,0366$ & $-47,9916$ & São Carlos & BU & 26 & $\mathrm{FF}$ \\
\hline 15 & $-21,8144$ & $-47,9572$ & São Carlos & $\mathrm{CX}$ & 15 & PAS \\
\hline 32 & $-21,7583$ & $-47,8250$ & São Carlos & TU & 7 & PAS \\
\hline
\end{tabular}

$\mathrm{ID}=$ identificação, $\mathrm{A}=$ artificial, $\mathrm{N}=$ natural, $\mathrm{MT}=$ maternidade, $\mathrm{MS}=$ abrigo de macho solteiro, $\mathrm{D}=$ digestório, $\mathrm{C}=$ casa, $\mathrm{CAB}=$ casa abandonada, $\mathrm{CX}=$ caixa de agua abandonada, $\mathrm{TU}=$ túnel, $\mathrm{G}=$ gruta, $\mathrm{PO}=$ porão, $\mathrm{TL}=$ tulha, $\mathrm{GP}=$ galeria de agua pluvial, $\mathrm{CR}$ = curral, $\mathrm{M}=$ moinho, $\mathrm{AG}=$ Agricultura Perene, $\mathrm{CSP}=$ Cultura Semi Perene, $\mathrm{FF}=$ Formação Florestal, $\mathrm{PAS}=$ Pastagens.

Tabela 6. Número e percentual de abrigos de Desmodus rotundus encontrados em suas respectivas declividades.

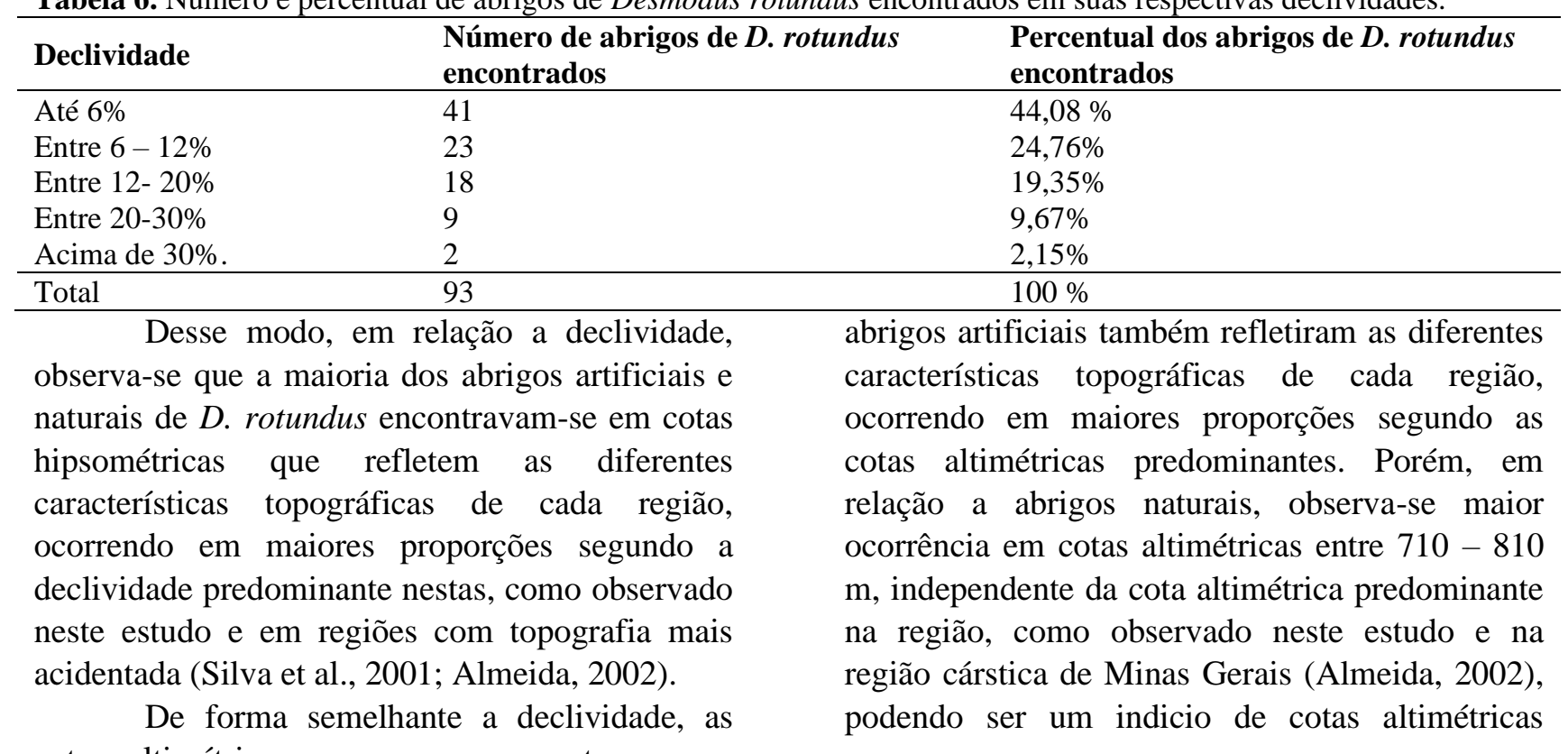


preferenciais para a colonização de abrigos naturais por D. rotundus.

Em relação ao uso do solo, a maior parte do total de abrigos de D. rotundus encontrados (51 abrigos, 54,83\%) estavam em áreas de agricultura, que somadas às áreas de cultura semi perene (15 abrigos, $16,12 \%$ ) totalizam $70,95 \%$ das áreas onde foram encontrados os abrigos de $D$. rotundus, percentual superior aos encontrados em áreas de formação florestal (16 abrigos, 17,20\%) e em áreas de pastagens (11 abrigos, 11,82\%).

Tabela 7. Cotas altimétricas, número e percentual de abrigos artificiais e naturais de Desmodus rotundus

\begin{tabular}{llcll}
\hline Cota altimétrica & $\begin{array}{l}\text { Número total de abrigos } \\
\text { artificiais de } \boldsymbol{D} \text {. rotundus } \\
\text { encontrados }\end{array}$ & $\begin{array}{l}\text { Percentual do total de } \\
\text { abrigos artificiais de } \boldsymbol{D} \text {. } \\
\text { rotundus encontrados }\end{array}$ & $\begin{array}{l}\text { Número de } \\
\text { abrigos naturais } \\
\text { de D. rotundus } \\
\text { encontrados }\end{array}$ & $\begin{array}{l}\text { Percentual de } \\
\text { abrigos naturais } \\
\text { de } \boldsymbol{D} \text {. rotundus } \\
\text { encontrados }\end{array}$ \\
\hline $412-512 \mathrm{~m}$ & 2 & $2,30 \%$ & 1 & $16,66 \%$ \\
$512-612 \mathrm{~m}$ & 30 & $34,48 \%$ & - & - \\
$612-712 \mathrm{~m}$ & 36 & $41,38 \%$ & - & - \\
$712-812 \mathrm{~m}$ & 11 & $12,65 \%$ & 4 & - \\
$812-912 \mathrm{~m}$ & 1 & $1,15 \%$ & - & $16,66 \%$ \\
$912-1012 \mathrm{~m}$ & 7 & $8,04 \%$ & 1 & $100 \%$ \\
\hline Total & 87 & $100 \%$ & 6 & \\
\hline
\end{tabular}

A maioria dos abrigos naturais (4 abrigos, $66,67 \%)$ também se concentraram nas áreas de agricultura e culturas semi perene e os demais (2 abrigos, 33,33\%) em áreas de pastagem, sendo que nenhum abrigo natural foi encontrado em áreas de formação florestal. Todos os abrigos encontrados em áreas de formação florestal eram artificiais, com predominância de túneis (11 abrigos, 68,75\%) e os demais abrigos consistiam de casa habitada, casa abandonada, porão e tulha, indicando um alto grau de antropização do meio rural e florestal da região, com aumento da oferta de abrigos artificiais para $D$. rotundus.

Os abrigos localizados nas áreas de pastagem eram na sua maioria (9 abrigos, $81,81 \%$ ) artificiais, sendo distribuídos de forma regular nas cotas altimétricas entre 412 a $812 \mathrm{~m}$. Apenas dois abrigos localizados nestas áreas eram naturais $(18,19 \%)$, um localizado entre $412-512$ $\mathrm{m}$ e outro na cota de $712-812 \mathrm{~m}$.

Dados recolhidos em todo o Brasil mostraram que as cavernas de morcegos hematófagos são realmente uma exceção, geralmente restritas a áreas com alta densidade de cavernas. Este é o caso de cavernas de arenito, tais como aqueles no Pará e São Paulo (Trajano e Moreira, 1991; Campanha e Fowler, 1993). A paisagem menos irregular em regiões de arenito é mais favorável à pecuária extensiva e agricultura (Trajano, 1996).

O relevo de cada região deve ser considerado como um condicionador para o tipo de cobertura e o do uso do solo, que por sua vez, atuariam como modeladores espaciais para a ocorrência de abrigos de D. rotundus e da raiva dos herbívoros. Grandes áreas de florestas, de forma geral, estão situadas nas regiões de maior declividade; já áreas extensivas de plantações e pastagens situam-se nas partes mais planas e média declividade (Gomes et al., 2011).

Desta forma, devido à alta adaptabilidade de $D$. rotundus a ambientes antropizados, os fatores topográficos relacionados à presença de abrigos artificiais destes morcegos não apresentaram cotas de declividade e altimétrica preferenciais. Embora a maioria dos abrigos artificiais (66 abrigos, 75,86\%) estarem em cotas altimétricas entre 512 - $712 \mathrm{~m}$, a localização destes abrigos artificiais nestas cotas reflete a topografia predominante na região (Figura 2).

Em relação aos fatores topográficos relacionados à presença de abrigos naturais de morcegos hematófagos, as cotas de declividade também estavam relacionadas com a topografia dominante da região, de forma semelhante ao que ocorreu em abrigos artificiais. Porém, em relação à altimetria, percebe-se que a maioria deles localizam-se preferencialmente em cotas altimétricas superiores a $710 \mathrm{~m}$, como observado tanto no presente estudo, que possui predominantemente baixa declividade e altitudes medias, assim como em regiões de alta declividade e altitudes elevadas (Almeida, 2002).

Estudos na região nordeste do estado de São Paulo mostram que as progressões da raiva bovina se aproximaram de áreas com baixa altimetria e declividade (várzeas dos rios) e ausência de grandes áreas florestais (Gomes et al., 2011), e trabalhos realizados em Minas Gerais (Silva et al., 2001) constataram que a raiva dos herbívoros esteve associada às lavouras permanentes e temporárias, características de 
paisagem semelhantes às encontradas na maioria dos abrigos de $D$. rotundus no presente estudo.

Apesar de áreas de pastagens devido à presença de bovinos estarem comumente associadas a modelos de áreas de risco para circulação do vírus da raiva em herbívoros (Gomes et al. 2007; Dias et al., 2011; Gomes et al, 2011), no presente estudo a minoria dos abrigos (11 abrigos, 11,82\%) estava em áreas de pastagens, sendo que dois abrigos eram naturais. Isso pode ser explicado devido ao menor número de construções rurais encontradas em áreas de pastagens em relação a áreas de agricultura e culturas semi perenes, pois estas últimas comumente apresentam maior quantidade e diversidade de construções (casas abandonadas, tulhas, túneis, galeria de água pluvial, etc.) que aumentam a oferta de abrigos artificiais para esses morcegos.

Devido a sua grande adaptabilidade a ambientes antropizados, podemos considerar $D$. rotundus como uma boa espécie indicadora de perturbação antrópica do habitat ou de alteração do uso da terra (Medellín, 1993).

Poucos estudos informam dados quantitativos a respeito da topografia em que encontram-se abrigos de morcegos hematófagos, sendo a maioria deles qualitativos. A quantificação desses dados é importante para estudos que avaliam os fatores de receptividade à presença de abrigos de D. rotundus, fatores estes que por sua vez costumam ser incluídos em modelos de áreas de risco de transmissão da raiva bovina.

O predomínio de abrigos de D. rotundus localizados em áreas de agricultura e de baixa declividade no estado de São Paulo de certa forma difere das observações do MAPA - Ministério da Agricultura, Pecuária e Abastecimento (BRASIL, 2009) ao se considerar que regiões declivosas e áreas com florestas são as mais propícias para o estabelecimento de abrigos de $D$. rotundus e o grassamento da raiva dos herbívoros (BRASIL, 2009; Gomes et al., 2011).

Em vista disto, faz-se necessária uma reavaliação dos fatores de receptividade à presença de abrigos de morcegos hematófagos relacionados à topografia e ao tipo e à cobertura do solo que são incluídos em modelos de áreas de risco de ataques de morcegos hematófagos a herbívoros domésticos, devendo-se levar em consideração os efeitos de escala de cada trabalho e as características de paisagem da região estudada.

\section{Conclusão}

A maioria dos abrigos de $D$. rotundus no presente estudo esteve associado a áreas menos declivosas de agricultura e culturas semi perenes, e nenhum abrigo natural foi encontrado em áreas de formação florestal, demonstrando intensa perturbação antrópica da região com aumento da oferta de abrigos artificiais para D. rotundus. A predominância da ocorrência de abrigos naturais em cotas altimétricas superiores a $710 \mathrm{~m}$ encontrados neste estudo e outros trabalhos com topografias diversas pode ser um indício de altitudes preferenciais à colonização de $D$. rotundus para esse tipo de abrigo. A quantificação dos fatores de receptividade à presença de abrigos de $D$. rotundus pode auxiliar na elaboração de modelos de áreas de risco de ataques de morcegos hematófagos a herbívoros domésticos.

\section{Conflito de Interesse}

Os autores declaram não existir conflito de interesse de ordem pessoal ou econômica no manuscrito.

\section{Comitê de Ética}

A obtenção dos dados precede a criação da Comissão de Ética no Uso de Animais na UFSCar - Universidade Federal de São Carlos, na qual foi criada pela portaria GR 721/2014 e Lei Federal n. 11794 de 8 de outubro de 2008, que regulamenta o uso de animais para pesquisa e também é posterior a obtenção dos dados. Não obstante, a pesquisa não envolveu a coleta de animais em campo, sendo realizada apenas a observação indireta.

\section{Referências}

Acha, P.N.; Syfres, B. Zoonoses and communicable disease common to man and animals. $3^{\mathrm{a}}$ ed. Washington: Pan American Health Organization, 2003. v. 2, 425 p. Disponível em: <https://www.paho.org/hq/dmdocuments/201 0/ZoonosesVol-2.pdf>. Acesso em 21 out. 2020.

Almeida, E.O.; Moreira, E.C.; Naveda, A.B.; Herrmann, G. P. Combate ao Desmodus rotundus rotundus (E. Geoffroy, 1810) na região cárstica de Cordisburgo e Curvelo, Minas Gerais. Arquivo Brasileiro de 
Medicina Veterinária e Zootecnia, 54 (2): 117-126, 2002.

Batista, H.B.C.R.B.; Franco, A.C.F.; Roehe, P.M. Raiva, uma breve revisão. Acta Scientiae Veterinariae, 35(2): 125-144, 2007.

Bolívar-Cimé, B.; Flores-Peredo, R.; GarcíaOrtíz, S.A.; Murrieta-Galindo, R.; Laborde, J. Influencia de la estructura del paisaje en la abundancia de Desmodus rotundus (Geoffroy 1810) en el noreste de Yucatán, México Ecosistemas y Recursos Agropecuarios. 6(17): 263-271, 2019.

BRASIL. Ministério da Agricultura, Pecuária e Abastecimento. Controle da raiva dos herbívoros: manual técnico. Brasília: Mapa/ACS, 2009. p. 43-56. Disponível em:

<http://www.agricultura.gov.br/arq_editor/file/An iamal/programa\%20nacional\%20dos

\%20herbivoros/revis\%C3\%A3o\%20sobre\%20raiv a.pdf >. Acesso em 21 out. 2020

Bredt, A.I.; Araújo, F.A.A.; Caetano-Júnior, R.; Rodrigues, M.G.R.; Yoshizawa, M.; Silva, M.M. S.; Harmani, N.M.S.; Massunaga, P.N.T.; Bürer, S.P.; Potro, V.A.R.; Uieda, W. Morcegos em áreas urbanas e rurais: manual de manejo e controle. Brasília: Fundação Nacional de Saúde/Ministério da Saúde, 1996, 117p.

Bredt, A.; Uieda, W.; Magalhães, E.D. Morcegos cavernícolas da região do Distrito Federal, centro-oeste do Brasil (Mammalia, Chiroptera). Revista Brasileira de Zoologia, 16(3): 731-770, 1999.

Campanha, R.A.C.; Fowler, H.G. Roosting assemblages of bats in arenitic caves in remnant fragments of Atlantic Forest in southeastern Brazil. Biotropica, 25(3): 362365, 1993

CDA-SP, Coordenadoria de Defesa Agropecuária de São Paulo 2016. São Paulo tem 4,6 mil abrigos de morcegos hematófagos cadastrados. Disponível em: $<$ https://www.defesa.agricultura.sp.gov.br/no ticias/2016/sao-paulo-tem-46-mil-abrigos-demorcegos-hematofagos-

cadastrados,975.html >. Acesso em 21 out. 2020.

Dias, R.A.; Nogueira Filho, V.S.; Goulart, C.S.; Telles, I.C.O.; Marques, G.H.F.; Ferreira, F.; Amaku, M.; Ferreira Neto, S. Modelo de risco para circulação do vírus da raiva em herbívoros no Estado de São Paulo, Brasil.
Revista Panamericana de Salud Publica, 30(4): 370-376, 2011.

Fisher, C.R.; Streicker, D.G., Schnell, M.J. The spread and evolution of rabies virus: conquering new frontiers. Nature Reviews Microbiology, 16(4): 241- 255, 2018.

Gomes, M.N.; Uieda, W. Abrigos diurnos, composição de colônias, dimorfismo sexual e reprodução do morcego hematófago Desmodus rotundus (E. Geoffroy) (Chiroptera, Phyllostomidae) no Estado de São Paulo, Brasil. Revista Brasileira de Zoologia 21(3): 629-638, 2004.

Gomes, M.N.; Monteiro, A.M.V.; Nogueira, V.S.; Gonçalves, C.A. Áreas propícias para o ataque de morcegos hematófagos Desmodus rotundus em bovinos na região de São João da Boa Vista, Estado de São Paulo. Pesquisa Veterinária Brasileira, 27(7): 307-313, 2007.

Gomes, M.N.; Monteiro, A.M.V; Escada, M.I.S. Raiva bovina segundo os mosaicos de uso e cobertura da terra no estado de São Paulo entre 1992 e 2003. Arquivo Brasileiro de Medicina Veterinária e Zootecnia, 63(2): 287-295, 2011.

Greenhall, A.M.; Joermann, G.; Schmidt, U.; Seidei, M.R. Desmodus rotundus. Mammalian Species, 202: 1-6, 1983.

Johnson, N.; Aréchiga-Ceballos, N.; AguilarSetien, A. Vampire bat rabies: Ecology, Epidemiology and Control. Viruses, 6: 19111928, 2014.

Lee, D.N.; Papes, M.; Denbussche, R.A.V. Present and potential future distribution of common vampire bats in the Americas and the associated risk to cattle. PLoS ONE, 7(8): e4266, 2012.

Medellín, R.A. Estructura y diversidad de una comunidad de murciélagos en el trópico húmedo mexicano. In: Medellín, R.A.; Ceballos, G. Avances en el estudio de los mamíferos de México. México: Asociación Mexicana de Mastozoología. Publicaciones especiales, 1993, p. 333-354.

Mialhe, P.J.; Moschini, L.E. Análise de fatores de receptividade e vulnerabilidade na elaboração de modelo de risco de ataques de morcegos hematófagos a bovinos no município de São Pedro - SP. Archives of Veterinary Science, 23(2): 72-83, 2018. 
Neuweiler, G.; Covey, E. The biology of bats. New York: Oxford University Press, 2000. 310p.

Oliveira, P.R.; Silva, D.A.R.; Rocha, J.H.; de Melo, S.M.A.; Bombonato, N.G.; Carneiro e Silva, F.O. Levantamento, cadastramento e estimativa populacional das habitações de morcegos hematófagos, antes e após atividades de controle, no Município de Araguari, MG. Arquivo do Instituto Biológico do Estado de São Paulo, 76(4): 553- 560, 2009.

SÃO PAULO. Secretaria de Agricultura e Abastecimento, Instituto de Economia Agrícola (IEA). Centro Leste Paulista Ganha Pólo Regional Dos Agronegócios. Disponível em: <http://www.iea.sp.gov.br/out/TerTexto.php? codTexto $=670>$. Acesso em 21 out. 2020.

Silva, J.A.; Moreira, E.C.; Haddad, J.P.A.; Sampaio, I.B.M.; Modena, C.M.; Tubaldini, M.A.S. Uso da terra como determinante da distribuição da raiva bovina em Minas Gerais, Brasil. Arquivo Brasileiro Medicina
Veterinária e Zootecnia, 53(3): 273-283, 2001.

Souza, P.G.; Amaral, B.M.P.M.; Gitti, C.B. Raiva animal na cidade do Rio de Janeiro: emergência da doença em morcegos e novos desafios para o controle. Revista do Instituto Adolfo Lutz, 73(1): 119-124, 2014. Taddei, V.A.; Gonçalves, C.A.; Pedro, W.A.; Tadei, W.J.; Kotai, I.; Arieta, C. Distribuição do morcego vampiro Desmodus rotundus no Estado de São Paulo e a raiva dos animais domésticos. Coordenadoria de Assistência Técnica Integral, Campinas, 1991. 107p.

Trajano, E. Movements of cave bats in Southeastern Brazil, with emphasis on the population ecology of the common vampire bat, Desmodus rotundus (Chiroptera) Biotropica, 28(1): 121-129, 1996.

Vigilato, M.A.N.; Cosivi, O.; Clavjo, Knöbl, T.A.; Silva, H.M.T. Rabies update for Latin America and the Caribbean. Emerging Infectious Diseases, 19(4): 678-679, 2013. 